\title{
Ultra-long-term lithium therapy: all-important matters and a case of successful 50-year lithium treatment
}

\author{
Ewa Ferensztajn-Rochowiak, (iD Maria Chłopocka-Woźniak, Janusz K. Rybakowski iD \\ Department of Adult Psychiatry, Poznan University of Medical Sciences, Poznan, Poland.
}

\begin{abstract}
This paper discusses essential issues related to long-term lithium therapy and presents a case of successful 50-year lithium treatment. Lithium is currently regarded as the drug of choice for preventing manic and depressive recurrences in bipolar disorder. In 1/3 of patients with bipolar disorder, long-term monotherapy with lithium can completely prevent recurrences of abnormal mood. Numerous clinical and psychosocial factors associated with a good response to lithium have been described. Lithium is more efficacious than other mood stabilizers, and its long-term treatment significantly exceeds them. Lithium also exerts antisuicidal, immunomodulatory, and neuroprotective effects. The main problems associated with long-term lithium treatment include kidney, thyroid, and probably cognitive issues. In this paper, a case of successful continuous lithium treatment for 50 years in a 79-year-old female patient is presented. In this patient, apart from maintaining a euthymic state, long-term lithium treatment also exerted a favorable effect on general health, especially the elimination of viral and other respiratory infections. It is concluded that ultra-long term lithium therapy can enable good professional and psychosocial functioning for many patients, and the possible somatic side effects are manageable.
\end{abstract}

Keywords: Mood disorders; bipolar; lithium

\section{Introduction}

Lithium is currently regarded as the first-choice mood stabilizer for maintenance treatment of bipolar disorder. This was first demonstrated in the early 1960s, and in the 21 st century the efficacy of prophylactic lithium has been convincingly confirmed. ${ }^{1-3}$ Compared to other mood stabilizers, lithium is more efficacious and also exerts antisuicidal, immunomodulatory, and neuroprotective effects. Although lithium has been used in significantly longerterm treatment than any other mood stabilizer, long-term therapy is not devoid of adverse effects, the most important of which is kidney damage.

\section{The quality of long-term lithium response}

Meta-analyses of the prophylactic efficacy of lithium in bipolar disorder in the 21st century can be shortly summarized. Geddes et al., ${ }^{1}$ based on five randomized controlled trials including 770 patients, found that lithium was significantly more effective than placebo for the prophylaxis of abnormal mood relapse, with somewhat better anti-manic than anti-depressive efficacy. Nivoli et al. $^{2}$ analyzed 1,561 patients (534 treated with lithium) who participated in controlled studies of at least sixmonths. They observed that more recent trials found more effective anti-manic than anti-depressive prophylactic

Correspondence: Janusz K. Rybakowski, Department of Adult Psychiatry, University of Medical Sciences, ul.Szpitalna, 27/33, 60572, Poznan, Poland.

E-mail: janusz.rybakowski@gmail.com

Submitted May 08 2020, accepted Jun 24 2020, Epub Sep 182020. efficacy for lithium, whereas older studies had suggested similar preventive effectiveness for both mania and depression. Severus et al. $^{3}$ analyzed seven studies with a total of 1,580 patients that compared lithium with placebo. They found that lithium was significantly better than placebo for the prophylaxis of abnormal mood relapse and manic episodes. Some trials also found that lithium had better preventive efficacy against depressive episodes than placebo.

The prophylactic effect of lithium on mood disorders was first demonstrated in the early $1960 \mathrm{~s},{ }^{4,5}$ and since then lithium has become the prototype "mood stabilizer." Mood stabilizers can be classified according to their introduction in psychiatry to prevent the relapse of mood disorders. First-generation mood stabilizers, such as lithium, valproates, and carbamazepine, first appeared in the 1960s and 1970s. Second-generation mood stabilizers, including atypical antipsychotics such as clozapine, olanzapine, quetiapine, aripiprazole and risperidone, and a new anti-epileptic drug, lamotrigine, have been introduced since the latter half of the 1990s. ${ }^{6,7}$

MAP (Multicentre study of long-term treatment of Affective and schizoaffective Psychoses) and BALANCE (Bipolar Affective disorder Lithium/ANtiConvulsant Evaluation) were important trials that compared lithium with other first-generation mood stabilizers. In the MAP trial, recurrences in patients with bipolar disorder were analyzed during 2.5 years of lithium or carbamazepine treatment. It
How to cite this article: Ferensztajn-Rochowiak E, ChłopockaWoźniak M, Rybakowski JK. Ultra-long-term lithium therapy: allimportant matters and a case of successful 50-year lithium treatment. Braz J Psychiatry. 2021;43:407-413. http://dx.doi.org/ 10.1590/1516-4446-2020-1111 
was found that lithium was better than carbamazepine in bipolar I, whereas both drugs had similar efficacy in bipolar II. Although lithium treatment resulted in fewer hospitalizations in patients without mood-incongruent delusions or psychiatric comorbidities, carbamazepine was more effective in the other patients. ${ }^{8}$ In the BALANCE trial, 330 bipolar patients were randomly allocated to monotherapy with lithium $(n=110)$, valproate $(n=110)$, or a combination of both drugs $(n=110)$, and were followed up for 2 years. A new affective episode occurred in $54 \%$ of the combined group, $59 \%$ of the lithium group, and $69 \%$ of the valproate group. Interestingly, depressive episode recurrence was lowest in the lithium group (32\%), followed by the combined group (35\%) and the valproate group (45\%). The results of this study indicated the superiority of lithium. ${ }^{9}$

Goodwin et al. compared the prophylactic efficacy of lithium vs. lamotrigine, a second-generation mood stabilizer, ${ }^{10}$ following up bipolar patients who received lithium, lamotrigine, or placebo double-blindly for 18 months. Both lithium and lamotrigine outperformed placebo for preventing affective episodes. Although lithium prevented mania better than lamotrigine, lamotrigine prevented depression better than lithium. Severus et al. ${ }^{3}$ compared the prophylaxis efficacy of lithium with anticonvulsants such as valproate, carbamazepine, and lamotrigine in a large group of 1,305 subjects, finding that lithium was superior to anticonvulsants for manic but not depressive episodes.

In a study called Bipolar CHOICE (The Clinical and Health Outcomes Initiative in Comparative Effectiveness for Bipolar Disorder), lithium was compared with quetiapine, another second-generation mood stabilizer. The 6-month multicenter study, in which both drugs were used in typical settings of clinical practice, found that they had similar clinical efficacy. ${ }^{11}$ Ketter et al. ${ }^{12}$ proposed that lithium and quetiapine may have similar efficacy for acute episodes and short-term prophylaxis of bipolar disorder. In such conditions, a combination of the drugs is significantly more efficacious than either agent alone. However, in a naturalistic study lasting four years, Altamura et al. ${ }^{13}$ found that the number of euthymic patients was higher among the lithium group $(18 / 39 ; 46 \%)$ than the quetiapine group (12/49; 29\%).

Kessing et al. ${ }^{14}$ compared lithium monotherapy and monotherapy with different mood stabilizers in observational studies, finding that, in real life, lithium was more effective than the most frequently used mood stabilizers, such as valproate, lamotrigine, olanzapine, and quetiapine.

Lithium has a prophylactic effect against depressive episodes, although it is probably slightly less than its effect against manic episodes. In his pivotal 1963 paper, Hartigan demonstrated that lithium has a prophylactic effect on both bipolar and unipolar mood disorder. ${ }^{4}$ Recently Finnish researchers showed that, of all pharmacological treatments, lithium monotherapy has the lowest risk of hospital readmission in patients with severe unipolar depression. ${ }^{15}$

\section{Clinical and psychosocial factors associated with favorable long-term lithium efficacy}

Patients with mood disorders who have benefitted the most from long-term lithium treatment have been referred to as "excellent lithium responders." The epithet was proposed by Canadian psychiatrist Paul Grof ${ }^{16}$ for patients whose lithium monotherapy caused a total disappearance of abnormal mood episodes for an extended period (e.g., $>10$ years). According to Grof, the clinical characteristics of these subjects would be an episodic clinical course with distinct periods of remission, a family history of bipolar disorder, and few or no psychiatric co-morbidities, which was similar to Emil Kraepelin's classic description of manic-depressive disorder in $1899 .{ }^{17}$ Approximately $30 \%$ of bipolar patients treated with lithium can be considered excellent lithium responders. When defined as patients without recurrences in 10 years of lithium monotherapy, the proportion of excellent lithium responders was comparable for patients who began lithium treatment at our center in the 1970s (34\%) and 1980s (27\%). ${ }^{18}$

In 2005, German researchers performed a meta-analysis of clinical factors related to prophylactic lithium response in bipolar disorder. As favorable clinical features, they identified an episodic pattern of mania-depression-interval and a later illness onset, whereas an episodic pattern of depression-mania-interval, continuous cycling, and a high number of previous hospitalizations were indicated as unfavorable features. ${ }^{19}$ These authors also analyzed psychosocial and demographic factors according to lithium response. Good outcome predictors included high social status, social support, good compliance, and a psychological tendency to dominate, whereas poor outcome predictors included susceptibility to stress, high expressed emotions, neurotic personality traits, unemployment, and a high number of life events. ${ }^{20}$

In Poznan, Poland, we assessed a group of 111 patients (25 male, 76 female) who received lithium for 5-39 (mean 18) years regarding clinical factors associated with the prophylactic efficacy of lithium. We found a better effect in patients with later illness onset, no family history of mood disorders, and family members on prophylactic lithium, as well as in women with comorbid anxiety disorders and men who did not abuse alcohol. ${ }^{21}$

Recently, British researchers performed a meta-analysis of 71 studies with a total of 12,000 patients regarding clinical factors associated with quality of prophylactic lithium response. They identified such positive features as a mania-depression-remission episode sequence, non-rapid-cycling, no psychotic episodes, a family history of bipolar disorder, short illness duration prior to lithium initiation, and later illness onset. Fewer episodes and hospitalizations prior to lithium treatment, as well as lithium treatment in other family members were also described as favorable factors. ${ }^{22}$

In a 2020 study, Nunes et al. ${ }^{23}$ assessed 1,266 lithiumtreated patients from seven European and Canadian centers. In agreement with previous results, the authors demonstrated that an episodic clinical course and nonrapid-cycling were the most significant factors for a good lithium response.

Therefore, an episodic clinical course with a maniadepression-remission sequence, later illness onset, nonrapid-cycling, and early lithium therapy have been repeatedly demonstrated as favorable factors for longterm lithium response. Our previous result ${ }^{21}$ that lithium is 
less efficacious in male patients who abuse alcohol was corroborated by O'Connell et al. ${ }^{24}$ Our finding of better lithium response in patients with family members who were also on prophylactic lithium corresponds with the good next generation response described by Grof. ${ }^{17}$ The relationship between lithium responsiveness and family history of mood disorders remains controversial.

We also studied the association between lithium response and personality features using the Temperament Evaluation of the Memphis, Pisa, Paris, and San Diego Autoquestionnaire (TEMPS-A) and the Oxford-Liverpool Inventory of Feelings and Experiences (O-LIFE). With the TEMPS-A, which assesses five affective temperaments (hyperthymic, cyclothymic, depressive, anxious, and irritable), we observed a positive correlation between long-term efficacy and a hyperthymic temperament, as well as a negative correlation with anxious, cyclothymic and irritable temperaments. ${ }^{25}$ This could correspond with the negative relationship between lithium prophylaxis and neurotic personality features reported in the previously mentioned German study. ${ }^{20}$ Using the O-LIFE scale, which assesses four dimensions of schizotypy (unusual experiences, introvertive anhedonia, cognitive disorganization, and impulsive nonconformity), we found a significant negative correlation with cognitive disorganization, which is associated with a predisposition to psychotic symptoms. ${ }^{26}$ This could correspond with a genetic study by the International Consortium of Lithium Genetics, which showed that the polygenic score for schizophrenia determines lower lithium responsiveness. ${ }^{27} \mathrm{~A}$ limitation of the above-mentioned studies is that they all consisted of retrospective clinical observations and the statistical analyses were mainly correlations.

\section{Adverse effects of long-term lithium treatment}

Four years ago, Gitlin ${ }^{28}$ reviewed lithium's side effects and toxicity and suggested strategies of their management. It seems that the main adverse effects of long-term treatment are generally related to thyroid, renal and cognitive function. Long-term side effects frequently associated with the thyroid gland include goiter and hypothyroidism. Recently, we studied 137 patients with bipolar disorder, including 98 (30 males) who had been treated with lithium for at least three years (mean $19 \pm 10$ years) and 39 (12 males) lithium-naive patients. The patients' age and illness duration were similar in both groups. Thyroid-stimulating hormone concentrations and thyroid gland volumes were significantly higher in patients receiving lithium. However, the frequency of hypothyroidism in the course of the illness was similar in both groups ( $24 \%$ vs. $18 \%$ ), which could show that bipolar illness itself can be a risk factor for hyperthyroidism. In both lithiumtreated and lithium-naive patients, the prevalence of hypothyroidism was 3-4 times higher in women than men. Subjects with hypothyroidism were successfully treated with levothyroxine. ${ }^{29}$ No connection was found between long-term lithium treatment and antithyroid antibodies. ${ }^{30}$ We also observed similar indexes of thyroid function in bipolar patients who had used lithium for 10-20 years and in those who had used it for 20 years or more. ${ }^{31}$
The most common renal adverse effect of lithium is decreased renal concentrating capacity, which can appear after a few weeks of lithium use. However, a more serious concern is the possibility of lithium-induced interstitial nephropathy, which can develop after 10-20 years of treatment and leads to increased creatinine concentration and a decreased glomerular filtration rate (GFR). In a recent international study (12 participating centers), the data of 312 bipolar disorder patients with a mean age of 56 (range 20-89) years who had received lithium carbonate for 8-48 (mean 18) years were analyzed. Nearly $1 / 3$ of the patients had a GFR value $<60 \mathrm{~mL} / \mathrm{min} / 1.73 \mathrm{~m}^{2}$, more frequently after $\geqslant 15$ years of lithium treatment and after 55 years of age. However, no case of end-stage renal failure was detected. The GFR reduction amounted to $0.71 \%$ with each year of age and $0.92 \%$ with each year of lithium treatment. Risk factors for declining GFR included longer lithium treatment, higher serum lithium concentrations, older age, medical co-morbidities, and a lower initial value when beginning lithium treatment after 40 years of age. ${ }^{32}$

We assessed kidney function during five-years of follow up in four good responders to lithium (three males and one female, aged 67-69 years, treated with lithium for $27 \pm 9$ years) whose GFR was $<50 \mathrm{~mL} / \mathrm{min} / 1.73 \mathrm{~m}^{2}$. During the five-year follow-up period, in three patients whose initial GRF was between $47-48 \mathrm{~mL} / \mathrm{min} / 1.73 \mathrm{~m}^{2}$, kidney parameters, such as GFR, serum creatinine, and urine specific gravity, did not significantly change. Therefore, in lithium responders whose GFR is not much lower than $50 \mathrm{~mL} / \mathrm{min} / 1.73 \mathrm{~m}^{2}$, we suggest continuing lithium with an annual checkup of renal parameters. In patients with a GFR of $32 \mathrm{~mL} / \mathrm{min} / 1.73 \mathrm{~m}^{2}$, the GFR decreased by $14 \%$ and serum creatinine increased by $10 \%$. In such patients, we recommend reducing the lithium dose and more frequent renal monitoring. ${ }^{33}$

In summary, progressive renal damage may occur in a small fraction of patients on long-term lithium therapy. In such cases, lithium is often discontinued and replaced with another mood stabilizer. However, the decision to discontinue lithium should be made with caution, especially in good responders, since other mood stabilizers may not be equally efficacious. In the majority of these patients, discontinuing lithium results in a high risk of relapse and further treatment resistance. Therefore, in patients with lithium-induced nephropathy, renal function should be closely and frequently monitored, and guidelines for managing such patients have recently been published. ${ }^{34}$

Some clinicians believe that lithium may cause impaired cognition. A meta-analysis of bipolar patients treated with lithium and patients treated with other medications showed that lithium had a moderately unfavorable effect on cognitive function. ${ }^{35}$ This is in contrast to experimental studies that found enhanced learning and memory with lithium. ${ }^{36-38}$ In a more recent clinical study, Spanish researchers found lower performance on some neuropsychological tests in bipolar patients than healthy subjects but no differences between bipolar patients receiving lithium or no medications. They concluded that lithium treatment had no deleterious effect on cognition. ${ }^{39}$ 
Bersani et al. ${ }^{40}$ compared 15 euthymic bipolar patients treated with lithium for more than a year, 15 matched patients receiving other mood stabilizers, and 15 matched controls. They found that bipolar patients had deficiencies in visual memory and executive function, although only patients on other mood stabilizers had reduced visual memory.

The Poznan study demonstrated that the efficacy of lithium prophylaxis could determine cognitive function status. When the Wisconsin Card Sorting Test was applied to excellent lithium responders, partial responders, and non-responders, it was found that non-responders performed significantly worse than the other lithium groups and healthy controls on most domains of the test. ${ }^{41}$ In a second study, performance on neuropsychological tests that measure spatial working memory and sustained attention was assessed. On all neuropsychological tests, excellent lithium responders performed better than the other lithium groups, and their results were no different from those of healthy controls. ${ }^{42}$ A recent review of the effects of lithium on neurocognitive function by Rybakowski incorporated these findings. ${ }^{43}$

Lithium's effects on cognition (and probably other systems) can be minimized by ensuring an appropriate concentration, i.e., avoiding overdose. Among our patients, some have been treated with lithium for 40 years or more, and we have adhered to the prophylactic guidelines put forward nearly four decades ago, keeping the serum lithium concentration between $0.5-0.8 \mathrm{mmol} / \mathrm{l} .{ }^{44}$ The mean lithium level in our cognition research was $0.65 \mathrm{mmol} / \mathrm{l}$ and, when required, the dose of lithium was reduced, resulting in a concentration of about $0.5 \mathrm{mmol} / \mathrm{l}$.

\section{Distinctive properties of lithium vs. other mood stabilizers}

Much research has shown that lithium exerts the greatest anti-suicidal, immunomodulatory, antiviral and neuroprotective effects of all mood stabilizers. The mortality of bipolar patients is 2-3 times that of the general population, mainly due to suicides. Müller-Oerlinghausen et al. ${ }^{45}$ compared mortality and suicidal behavior in 471 patients during the initial and later period of lithium treatment. In the initial period, mortality was twice that of the general population and suicides were significantly more frequent. In the latter period, both mortality and suicides were similar to the general population. The authors concluded that the full anti-suicidal effect of lithium comes after two years of treatment. A recent meta-analysis by Cipriani et al. ${ }^{46}$ with 6,674 patients concluded that lithium was significantly better than placebo at reducing the number of suicides and deaths from any cause. In recurrent depression, lithium was also connected with a lower risk of suicide and fewer total deaths. As an anti-suicidal drug, lithium was found to be superior to other mood stabilizers or antidepressants.

Lithium can mitigate immune-endocrine components of bipolar disorder pathogenesis, such as acute-phase reaction, pro-inflammatory cytokine production, and excessive activation of the hypothalamic-pituitary-adrenal axis. ${ }^{47}$
Recently, we examined the impact of long-term lithium treatment on very small embryonic-like stem cells (VSELs) and mRNA expression of pluripotency and glial markers in peripheral blood. Bipolar patients not treated with lithium had more VSELs (according to illness duration) and increased expression of markers than matched healthy subjects. Patients treated with lithium had fewer VSELs and lower expression of some markers than those not treated with lithium, which suggests that lithium might alleviate excessive regenerative and inflammatory processes in bipolar disorder. ${ }^{48}$

Lithium can exert antiviral effects against herpes viruses. In our study, it was demonstrated that long-term treatment with lithium resulted in the disappearance of or greatly diminished recurrences of labial herpes caused by herpes simplex virus type $1 .{ }^{49}$ This could be related to the effect of lithium on cognition in bipolar patients and Alzheimer's disease. Infection with herpes simplex virus type 1 is correlated with the features of impaired cognition in bipolar illness, ${ }^{50}$ and herpes infection has also been found to be one of the most significant pathogenic factors in Alzheimer's disease. ${ }^{51}$

The neuroprotective effects of lithium were recently reviewed by Rybakowski et al. ${ }^{52}$ Lithium treatment has led to increased volume in some brain structures, but such an effect was not observed in patients treated with other mood stabilizers. ${ }^{53,54}$ Epidemiological research suggests that long-term lithium use may reduce the risk of dementia, both in the general population and in patients with bipolar disorders. Such a relationship has only been found with lithium; an elevated risk of dementia, which increases concomitantly with treatment duration, has been found in anticonvulsants, antidepressants, and antipsychotics. ${ }^{55,56} \mathrm{~A}$ meta-analysis of three studies suggested that lithium treatment also has some benefits for Alzheimer's disease and mild cognitive impairment. ${ }^{57}$

\section{Ultra-long-term lithium therapy}

Four years ago, we described five patients (two men, aged 64 and 79 years and three women, aged 64, 65, and 75 years) who had taken lithium for 40 years or more. We assessed their kidney, thyroid, and other functions with respect to the course of the disease and psychosocial functioning. Four of the patients were good or excellent lithium responders. In the 65-year-old female patient, despite recurrences and hospitalizations, the overall effect of long-term lithium treatment could be classified as relatively good. ${ }^{58}$

From a diagnostic point of view, four of the patients can be considered bipolar I, including severe mania episodes that require hospitalization. The 75-year-old female patient could be considered bipolar II, or bipolar spectrum, due to a hyperthymic personality and probable hypomania after her mother's death. As mentioned previously, a hyperthymic personality is a favorable factor for the long-term prophylactic efficacy of lithium. ${ }^{25}$ In addition, before starting lithium, the patient had frequent depressive episodes and, according to some authors, such multi-episodic depressive patients may belong to the bipolar spectrum. ${ }^{59}$ 
The course of the illness was clearly periodic in all of the patients, with distinct intervals of remission between episodes. No comorbid conditions, such as an anxiety disorder or substance abuse, occurred in any of them. Such a periodic course with distinct periods of remission and a lack of co-morbid conditions have been regarded as favorable factors for a good long-term response to lithium. ${ }^{17}$

Lithium treatment began within the first 1-2 years of illness in three of the patients, after 5 years in one, and after 7 years in the other. Franchini et al. ${ }^{60}$ suggest that early lithium treatment (within the first ten years after illness onset is a predictor of a good long-term outcome. In addition, Kessing et al. ${ }^{61}$ showed that early lithium treatment following first psychiatric contact or a single manic/mixed episode is associated with an increased probability of good response. Several studies have found that later illness onset may be a factor for a good response to lithium. ${ }^{19,22}$ This was the case in the 79-yearold male patient, whose illness began at 35 years of age.

The serum lithium concentration has been maintained at a moderate level in all five patients. This concentration was $0.60-0.65 \mathrm{mmol} / \mathrm{l}$ in four patients throughout the study, a level recommended several decades ago for minimizing the possible side effects of lithium. ${ }^{44}$ In the 79-year-old male patient, despite a low lithium carbonate dose $(750 \mathrm{mg} / \mathrm{d})$, the serum lithium level was between 0.7-0.8 mmol/l, although without significant somatic side effects.

Regarding renal function results, the 64-year-old male patient had stage 3 chronic kidney disease, and the kidney impairment reached stage $2 / 3$ in the other male patient. However, there were no signs of progressive renal damage in the last five years in these patients. In contrast, all three women had asymptomatic stage 2 chronic kidney disease. Previously, in an analysis of 80 long-term lithium-treated patients, we found worse kidney function parameters in men than in women. ${ }^{62}$ On the other hand, the results of Shine et al. ${ }^{63}$ could indicate that women are at higher risk of developing kidney damage during long-term lithium treatment than men.

Many studies have suggested that female bipolar patients in general are more prone to developing thyroid dysfunction. ${ }^{64}$ In our sample, the 65-year-old female patient had severe thyroid dysfunction (Hashimoto disease) with extremely high levels of anti-thyroid peroxidase and anti-thyroglobulin antibodies, for which she has been receiving thyroxine. The levels of thyroid hormones and antibodies in the four remaining patients were in the normal range.

In all patients, the preservation of cognitive functions was satisfactory. This enabled them to continue their professional activity until 55-65 years of age. This is in line with other studies by our group that found preserved cognitive function in good lithium responders. ${ }^{41,42}$

\section{Fifty years of lithium treatment}

Given that it is the 50th anniversary of lithium treatment for one of the patients in the above-mentioned study, her case will be described here in greater detail. This female patient, born in 1941, can be described as hyperthymic. She has always been optimistic and had good interpersonal relationships. She graduated from the Poznan Medical Academy in 1961. In 1963 she married, and in 1967 gave birth to a daughter. In 1973 she completed a specialization course in ophthalmology.

From the beginning of the 1960s, she suffered from labial herpes on average once a year. In 1966 she had shingles, and in 1968 was diagnosed with Wilson's lichen planus. Her first depressive episode appeared in 1965 without apparent cause and receded after 2 months of treatment with imipramine. The next depressive episodes occurred in 1968 and at the end of 1970. She was again treated with imipramine with good results. After the third depressive episode (April 1970), lithium carbonate treatment began and has continued without interruption until today. For the first 20 years, the dose was $1,000 \mathrm{mg} / 24 \mathrm{~h}$, taken once during the evening, and the mean lithium concentration in the serum was $0.6 \mathrm{mmol} / \mathrm{l}$. This dose was reduced to $750 \mathrm{mg} / 24 \mathrm{~h}$ in 1990 , to $500 \mathrm{mg} /$ day in 2005, and to $250 \mathrm{mg} /$ day in 2010 . Her mental state has been stable and she has not shown any significant fluctuations throughout the entire period of lithium treatment. In 1985 , after the death of her mother, a two-week period of increased emotional instability, likely hypomania, was observed, but this did not require additional pharmacological intervention. The patient worked successfully as an ophthalmologist until 2007.

During the lithium treatment period, labial herpes occurred only twice and she reported no colds or other viral infections. Examination of kidney function in 2012 revealed asymptomatic stage 2 chronic kidney disease with serum creatinine $0.97 \mathrm{mg} / \mathrm{dL}$, eGFR $60 \mathrm{~mL} / \mathrm{min} /$ $1.73 \mathrm{~m}^{2}$, and a calcium level of $2.44 \mathrm{mg} / \mathrm{dL}$. Regarding thyroid function, all hormones and antibodies assessed in 2013 were in the normal range. Since 2015, she has been treated for hypertension with cilazapril, $0.5 \mathrm{mg} /$ day.

This patient could be considered the epitome of an excellent lithium responder. Among the factors that predisposed her to a good response, we point out her hyperthymic personality ${ }^{25}$ and early lithium treatment (5 years after onset). ${ }^{59,60}$. It is especially important to point out the favorable effect of long-term lithium treatment on the general health of this patient, especially the elimination of viral and other respiratory infections. In our retrospective study we demonstrated that, in patients with mood disorders and recurrent labial herpes infections, lithium exerts an antiviral action, preventing or reducing the recurrence of labial herpes. ${ }^{49}$ We also observed a reduction in the mean rate of reported flu-like illness during lithium therapy. ${ }^{65}$

\section{Concluding remarks}

All-important issues of longitudinal lithium treatment were discussed with a special focus on the possibility of ultralong term lithium treatment. Such an extended duration (i.e., forty years or more) has not yet been matched by any other mood-stabilizing drug. The final section of the paper described a case of successful lithium treatment for 50 years. It can be concluded that ultra-long term lithium 
therapy is possible and enables good professional and psychosocial functioning in many patients. In such patients, the possible somatic side effects can be successfully managed.

\section{Disclosure}

The authors report no conflicts of interest.

\section{References}

1 Geddes JR, Burgess S, Hawton K, Jamison K, Goodwin GM. Longterm lithium therapy for bipolar disorder: systematic review and metaanalysis of randomized controlled trials. Am J Psychiatry. 2004; 161:217-22.

2 Nivoli AM, Murru A, Vieta E. Lithium: still a cornerstone in the longterm treatment in bipolar disorder? Neuropsychobiology. 2010; 62:27-35.

3 Severus E, Taylor MJ, Sauer C, Pfennig A, Ritter P, Bauer M, et al. Lithium for prevention of mood episodes in bipolar disorders: systematic review and meta-analysis. Int J Bipolar Disord. 2014;2:15

4 Hartigan GP. The use of lithium salts in affective disorders. $\mathrm{Br} \mathrm{J}$ Psychiatry. 1963;109:810-4.

5 Baastrup PC. The use of lithium in manic-depressive psychoses. Compr Psychiatry. 1964;5:396-408.

6 Rybakowski JK. Two generations of mood stabilizers. Int J Neuropsychopharmacol. 2007;10:709-11.

7 Rybakowski JK. Meaningful aspects of the term 'mood stabilizer'. Bipolar Disord. 2018;20:391-2.

8 Kleindienst N, Greil W. Differential efficacy of lithium and carbamazepine in the prophylaxis of bipolar disorder: results of the MAP study. Neuropsychobiology. 2000;42 Suppl 1: 2-10.

9 BALANCE investigators and collaborators; Geddes JR, Goodwin GM, Rendell J, Azorin JM, Cipriani A, et al. Lithium plus valproate combination therapy versus monotherapy for relapse prevention in bipolar I disorder (BALANCE): a randomised open-label trial. Lancet. 2010; 375:385-95.

10 Goodwin GM, Bowden CL, Calabrese JR, Grunze H, Kasper S, White R. A pooled analysis of 2 placebo-controlled 18-month trials of lamotrigine and lithium maintenance in bipolar I disorder. J Clin Psychiatry. 2004;65:432-41.

11 Nierenberg AA, McElroy SL, Friedman ES, Ketter TA, Shelton RC, Deckersbach T, et al. Bipolar CHOICE (Clinical Health Outcomes Initiative in Comparative Effectiveness): a pragmatic 6-month trial of lithium versus quetiapine for bipolar disorder. J Clin Psychiatry. 2016; 77:90-9.

12 Ketter TA, Miller S, Dell'Osso B, Wang PW. Treatment of bipolar disorder: review of evidence regarding quetiapine and lithium. J Affect Disord. 2016;191:256-73.

13 Altamura AC, Mundo E, Dell'Osso B, Tacchini G, Buoli M, Calabrese JR. Quetiapine and classical mood stabilizers in the long-term treatment of bipolar disorder: a 4-year follow-up naturalistic study. J Affect Disord. 2008;110:135-41.

14 Kessing LV, Bauer M, Nolen WA, Severus E, Goodwin GM, Geddes $\mathrm{J}$. Effectiveness of maintenance therapy of lithium vs other mood stabilizers in monotherapy and in combinations: a systematic review of evidence from observational studies. Bipolar Disord. 2018 Feb 14. doi: $10.1111 /$ bdi.12623. Online ahead of print.

15 Tiihonen J, Tanskanen A, Hoti F, Vattulainen P, Taipale H, Mehtala J, et al. Pharmacological treatment and risk of readmission to hospital for unipolar depression in Finland: a nationwide cohort study. Lancet Psychiatry. 2017;4:547-53.

16 Grof P. Excellent lithium responders: people whose lives have been changed by lithium prophylaxis. In: Birch NJ, Gallicchio VS, Becker RW, editors. Lithium: 50 years of psychopharmacology, new perspectives in biomedical and clinical research. Cheshire: Weidner Publishing Group; 1999. p.36-51.

17 Grof P. Sixty years of lithium responders. Neuropsychobiology. 2010;62:8-16

18 Rybakowski JK, Chłopocka-Woźniak M, Suwalska A. The prophylactic effect of long-term lithium administration in bipolar patients entering treatment in the 1970s and 1980s. Bipolar Disord. 2001; 3:63-7.

19 Kleindienst N, Engel RR, Greil W. Which clinical factors predict response to prophylactic lithium? A systematic review for bipolar disorders. Bipolar Disord. 2005;7:404-17.

20 Kleindienst N, Engel RR, Greil W. Psychosocial and demographic factors associated with response to prophylactic lithium: a systematic review for bipolar disorders. Psychol Med. 2005;35:1685-94.

21 Dembinska-Krajewska D, Kliwicki S, Chłopocka-Wożniak M, Rybakowski J. [The effectiveness of prophylactic use of lithium in bipolar disorder and schizotypal traits]. Pharmacother Psychiatr Neurol. 2012;28:153-8.

22 Hui TP, Kandola A, Shen L, Lewis G, Osborn DP, Geddes JR, et al. A systematic review and meta-analysis of clinical predictors of lithium response in bipolar disorder. Acta Psychiatr Scand. 2019;140:94-115.

23 Nunes A, Ardau R, Berghöfer A, Bocchetta A, Chillotti C, Deiana V, et al. Prediction of lithium response using clinical data. Acta Psychiatr Scand. 2020;141:131-41.

24 O'Connell RA, Mayo JA, Flatow L, Cuthbertson B, O'Brien BE. Outcome of bipolar disorder on long-term treatment with lithium. $\mathrm{Br} \mathrm{J}$ Psychiatry. 1991;159:123-9.

25 Rybakowski JK, Dembinska D, Kliwicki S, Akiskal KK, Akiskal HH. TEMPS-A and long-term lithium response: positive correlation with hyperthymic temperament. J Affect Disord. 2013;145:187-9.

26 Dembinska-Krajewka D, Kliwicki S, Chlopocka-Wozniak M, Rybakowski J. [The effectiveness of prophylactic use of lithium in bipolar disorder and schizotypal traits]. Pharmacother Psychiatry Neurol. 2012;28:153-8.

27 International Consortium on Lithium Genetics (ConLi+Gen); Amare AT, Schubert KO, Hou L, Clark SR, Papiol S, et al. Association of polygenic score for schizophrenia and HLA antigen and inflammation genes with response to lithium in bipolar affective disorder: a genome-wide association study. JAMA Psychiatry. 2018;75:65-74.

28 Gitlin M. Lithium side effects and toxicity: prevalence and management strategies. Int J Bipolar Disord. 2016;4:27.

29 Kraszewska A, Ziemnicka K, Jończyk-Potoczna K, Sowiński J, Rybakowski JK. Thyroid structure and function in long-term lithiumtreated and lithium-naïve bipolar patients. Hum Psychopharmacol. 2019;34:e2708.

30 Kraszewska A, Ziemnicka K, Sowiński J, Ferensztajn-Rochowiak E, Rybakowski JK. No connection between long-term lithium treatment and antithyroid antibodies. Pharmacopsychiatry. 2019;52:232-6.

31 Kraszewska A, Chlopocka-Wozniak M, Abramowicz M, Sowinski J, Rybakowski JK. A cross-sectional study of thyroid function in 66 patients with bipolar disorder receiving lithium for 10-44 years. Bipolar Disord. 2015;17:375-80.

32 Tondo L, Abramowicz M, Alda M, Bauer M, Bocchetta A, Bolzani L, et al. Long-term lithium treatment in bipolar disorder: effects on glomerular filtration rate and other metabolic parameters. Int $\mathrm{J}$ Bipolar Disord. 2017;5:27.

33 Abramowicz A, Permoda-Osip A, Nowak B, Olejniczak P, Rybakowski JK. Five-year observation of chronic renal insufficiency during lithium treatment. A case study of four patients. Pharmacother Psychiatry Neurol. 2017;33:169-79.

34 Severus E, Bauer M. Managing the risk of lithium-induced nephropathy in the long-term treatment of patients with recurrent affective disorders. BMC Med. 2013;11:34.

35 Wingo AP, Wingo TS, Harvey PD, Baldessarini RJ. Effect of lithium on cognitive performance: a meta-analysis. J Clin Psychiatry. 2009;70:1588-97.

36 Yazlovitskaya EM, Edwards E, Thotala D, Fu A, Osusky KL, Whetsell WO, et al. Lithium treatment prevents neurocognitive deficit resulting from cranial irradiation. Cancer Res. 2006;66:11179-86.

37 Nocjar C, Hammonds MD, Shim SS. Chronic lithium treatment magnifies learning in rats. Neuroscience. 2007;150:774-88.

38 Zhang L, Chen X, Feng W, Cui Y, Xu S, Che Y. Enhancing effects of chronic lithium treatment on detour learning in chicks. Biol Trace Elem Res. 2012;148:38-43.

39 Lopez-Jaramillo C, Lopera-Vasquez J, Ospina Duque J, García J, Gallo A, Cortez V, et al. Lithium treatment effects on the neuropsychological functioning of patients with bipolar I disorder. J Clin Psychiatry. 2010;71:1055-60.

40 Bersani G, Quartini A, Zullo D, lannitelli A. Potential neuroprotective effect of lithium in bipolar patients evaluated by neuropsychological 
assessment: preliminary results. Hum Psychopharmacol. 2016; 31:19-28.

41 Rybakowski JK, Permoda-Osip A, Borkowska A. Response to prophylactic lithium in bipolar disorder may be associated with a preservation of executive cognitive functions. Eur Neuropsychopharmacol. 2009;19:791-5.

42 Rybakowski JK, Suwalska A. Excellent lithium responders have normal cognitive functions and plasma BDNF levels. Int $\mathrm{J}$ Neuropsychopharmacol. 2010;13:617-22.

43 Rybakowski JK. Effect of lithium on neurocognitive functioning. Curr Alzheimer Res. 2016;13:887-93.

44 Schou M. Problems of lithium prophylaxis: efficacy, serum lithium, selection of patients. Bibl Psychiatr. 1981;160:30-7.

45 Müller-Oerlinghausen B, Wolf T, Ahrens B, Schou M, Grof E, Grof P, et al. Mortality during initial and during later lithium treatment. A collaborative study by the international group for the study of lithium-treated patients. Acta Psychiatr Scand. 1994;90:295-7.

46 Cipriani A, Hawton K, Stockton S, Geddes JR. Lithium in the prevention of suicide in mood disorders: updated systematic review and meta-analysis. BMJ. 2013;346: $\{3646$.

47 Rybakowski JK. Antiviral and immunomodulatory effect of lithium. Pharmacopsychiatry. 2000;33:159-64.

48 Ferensztajn-Rochowiak E, Kucharska-Mazur J, Tarnowski M, Samochowiec J, Ratajczak MZ, Rybakowski JK. Stem cells, pluripotency and glial cell markers in peripheral blood of bipolar patients on long-term lithium treatment. Prog Neuropsychopharmacol Biol Psychiatry. 2018;80:28-33.

49 Rybakowski JK, Amsterdam JD. Lithium prophylaxis and recurrent labial herpes infections. Lithium. 1991;2:43-7.

50 Dickerson FB, Boronow JJ, Stallings C, Origoni AE, Cole S, Krivogorsky $B$, et al. Infection with herpes simplex virus type 1 is associated with cognitive deficits in bipolar disorder. Biol Psychiatry. 2004;55:588-93

51 Itzhaki RF. Corroboration of a major role for herpes simplex virus type 1 in Alzheimer's disease. Front Aging Neurosci. 2018;10:324.

52 Rybakowski JK, Suwalska A, Hajek T. Clinical perspectives of lithium's neuroprotective effect. Pharmacopsychiatry. 2018;51:194-9.

53 Lyoo K, Dager SR, Kim JE, Yoon SJ, Friedman SD, Dunner DL, et al. Lithium-induced grey matter volume increase as a neural correlate of treatment response in bipolar disorder: a longitudinal brain imaging study. Neuropsychopharmacology. 2010;35:1743-50.

54 Germana C, Kempton MJ, Sarnicola A, Christodoulou T, Haldane M, Hadjulis $M$, et al. The effects of lithium and anticonvulsants on brain structure in bipolar disorder. Acta Psychiatr Scand. 2010; 122:481-7.

55 Kessing LV, Sondergard L, Forman JL, Andersen PK. Lithium treatment and the risk of dementia. Arch Gen Psychiatry. 2008;65:1331-5.

56 Kessing LV, Forman JL, Andersen PK. Does lithium protect against dementia? Bipolar Disord. 2010;12:87-94.

57 Matsunaga S, Kishi T, Annas $\mathrm{P}$, Basun $\mathrm{H}$, Hampel $\mathrm{H}$, Iwata $\mathrm{N}$. Lithium as a treatment for Alzheimer's disease: a systematic review and meta-analysis. J Alzheimers Dis. 2015;48:403-10.

58 Permoda-Osip A, Abramowicz M, Kraszewska A, Suwalska A, Chlopocka-Wozniak M, Rybakowski JK. Kidney, thyroid and other organ functions after 40 years or more of lithium therapy: a case series of five patients. Ther Adv Psychopharmacol. 2016;6: 277-82.

59 Saggese JM, Lieberman DZ, Goodwin FK. The role of recurrence and cyclicity in differentiating mood disorder diagnoses. Prim Psychiatry. 2006;13:43-51.

60 Kessing LV, Vradi E, Andersen PK. Starting lithium prophylaxis early v. late in bipolar disorder. Br J Psychiatry. 2014;205:214-20.

61 Franchini L, Zanardi R, Smeraldi E, Gasperini M. Early onset of lithium prophylaxis as a predictor of good long-term outcome. Eur Arch Psychiatry Clin Neurosci. 1999;249:227-30.

62 Rybakowski J, Abramowicz M, Drogowska J, Chlopocka-Wozniak M, Michalak M, Czekalski S. Screening for the markers of kidney damage in men and women on long-term lithium treatment. Med Sci Monit. 2012;18:CR656-60.

63 Shine B, McKnight RF, Leaver L, Geddes JR. Long-term effects of lithium on renal, thyroid, and parathyroid function: a retrospective analysis of laboratory data. Lancet. 2015;386:461-8.

64 Bauer M, Glenn T, Pilhatsch M, Pfennig A, Whybrow PC. Gender differences in thyroid system function: relevance to bipolar disorder and its treatment. Bipolar Disord. 2014;16:58-71.

65 Amsterdam JD, García-España F, Rybakowski J. Rates of flu-like infection in patients with affective illness. J Affect Disord. 1998; 47:177-82. 